\title{
MHD activity at the beta limit in RI mode discharges on TEXTOR-94
}

\author{
H.R. Koslowski ${ }^{\mathrm{a}}$, G. Fuchs ${ }^{\mathrm{a}}$, R. Jaspers ${ }^{\mathrm{b}}$, A. Krämer-Flecken ${ }^{\mathrm{a}}$, \\ A.M. Messiaen ${ }^{c}$, J. Ongena ${ }^{c}$, J. Rapp ${ }^{\mathrm{a}}$, F.C. Schüller ${ }^{\mathrm{b}}$, M.Z. Tokar’a \\ Trilateral Euregio Cluster: \\ a Institut für Plasmaphysik, Forschungszentrum Jülich GmbH, Euratom Association, \\ Jülich, Germany \\ b FOM Instituut voor Plasmafysica Rijnhuizen, Euratom Association, Nieuwegein, \\ Netherlands \\ ${ }^{c}$ Laboratoire de Physique des Plasmas/Laboratorium voor Plasmafysica, ERM/KMS, \\ Association Euratom-Belgian State, Brussels, Belgium
}

\begin{abstract}
The $\beta$ limit in radiative improved (RI mode) discharges where energy confinement is enhanced due to an increased radiation level from the edge by impurity seeding of neon has been investigated. Under these discharge conditions spontaneous confinement transitions from higher to lower energy confinement can be observed when the $\beta$ limit is approached. The empirically determined limit of the normalized toroidal beta, $\beta_{N}$, is $2.2, \beta_{p}$ reaches values up to 1.5 in the circular limiter tokamak TEXTOR-94. The sawtooth activity is often stabilized and replaced by central mode oscillations around $q=1$ when high values $(\geq 1)$ of the confinement enhancement factor $f_{H 93}$ with respect to the ELM-free ITER-93H mode scaling are reached. Measurements of the plasma current distribution show that already before the confinement transition the central current density decreases, i.e. the current profile broadens and magnetic shear is decreased. The deterioration of energy confinement has been found to correlate with the onset of MHD activity either at the $q=1.5$ and/or the $q=2$ surface. The enhanced transport after the $\beta$ drop is caused by the MHD mode activity in the plasma; the favourable stabilization of the ITG mode under RI mode conditions is not altered. The possible role of pressure driven contributions to the destabilization of the observed MHD modes will be discussed.
\end{abstract}

\section{Introduction}

Confinement studies on present day tokamaks have revealed that the maximum achievable value of normalized toroidal beta

$\beta_{N}=\beta_{t}(\%) /\left\{I_{p}(\mathrm{MA}) /\left[a(\mathrm{~m}) B_{t}(\mathrm{~T})\right]\right\}$

with

$\beta_{t}=2 \mu_{0}\langle p\rangle / B_{t}^{2}$

is limited below the prediction of ideal MHD theory [1] when it is tried to sustain high confinement for many confinement times. Only in transient discharges has this limit been reached. Resistive tearing modes with low poloidal and toroidal mode numbers, $m$ and $n$, respectively, have been found to be responsible for the limitation of energy confinement. These modes are so-called neoclassical modes because they grow although the tearing parameter $\Delta^{\prime}$ is negative, i.e. stabilizing. The destabilizing effect of these modes is due to the perturbed bootstrap current in the island. An overview of the theory on neoclassical tearing modes as well as experimental results from various tokamak experiments can be found in Refs [2-6]. One important feature of a neoclassical mode is that it requires a seed perturbation to grow. This seed island can be produced either by sawtooth crashes or by $m=1$ oscillations in the plasma core [7].

The radiative improved mode (RI mode), which has been discovered recently on the TEXTOR94 tokamak, is an attractive confinement regime which combines high energy confinement, high densities at or above the Greenwald density and a large fraction $(>50 \%)$ of radiative power exhaust caused by feedback controlled seeding of neon under quasi-stationary conditions [8]. For this confinement scheme enhancement factors $f_{H 93}$ with respect to ELM-free $\mathrm{H}$ mode scaling in excess of one have been reached. In this article $\beta$-limit studies for this confinement regime are discussed. Earlier experiments have shown that for plasma conditions with the highest enhancement factor values $\left(f_{H 93}>1\right)$ spontaneous confinement back-transitions, i.e. transitions 
from high to lower confinement, do occur. The observed confinement back-transitions are a soft limit, i.e. they reduce the energy in the plasma but do not generally lead to a disruption. The corresponding decrease in stored energy has been simulated by an exponential decay caused by a sudden change of the energy confinement time $\tau_{E}[9]$. The reason for this behaviour was still unclear, and a series of dedicated experiments to explore the underlying physical mechanisms of the confinement deterioration has been performed. In particular, it has been investigated if the confinement back-transition could be related to MHD activity and if this behaviour could be described by the theory of neoclassical tearing modes (NTMs).

The experiments were carried out on TEXTOR94 , a circular shaped tokamak $(R=1.75 \mathrm{~m}, a=$ $0.46 \mathrm{~m}$ ) equipped with a toroidal belt limiter. The plasma can be heated by two neutral beam injectors, one injecting in the direction of the plasma current (co-NBI), the other in the reverse direction (counterNBI). Each neutral beam injector can deliver a heating power up to $1.5 \mathrm{MW}$, which can be adjusted by either changing the acceleration voltage in the ion source or by partly closing an aperture in the beam line. Radiofrequency heating of the plasma is provided by two ICRH systems, each with a power of up to $2 \mathrm{MW}$.

Standard plasma operation is at a toroidal magnetic field $B_{t}=2.25 \mathrm{~T}$ with a plasma current $I_{p}=350 \mathrm{kA}$. The plasma conditions for the exploration of the $\beta$ limit were partly chosen to achieve a high $\beta_{N}$, i.e. using a lower magnetic field which was required to adapt the ICRH system to a lower operating frequency. In order to obtain a large poloidal beta, $\beta_{p}$, some of the experiments were performed at low plasma current, i.e. at high values of the edge safety factor $q_{a}$.

Most of the experiments for the evaluation of the $\beta$ limit have been conducted with balanced NBI, i.e. using co- and counter-injection in order to balance the momentum transfer to the plasma and keep the toroidal rotation speed low. As has been shown in Ref. [10] the slowing down of the rotation has only a minor, or even negligible, effect on the confinement quality of the discharge, thus proving that rotational shear is not the dominating factor for the improved confinement. This small toroidal rotation leads to a decrease of the observed mode frequencies; thus most of the applied diagnostics with sampling rates of $f_{\text {samp }}=20 \mathrm{kHz}$ were able to resolve the mode oscillations. When only neutral beam co-injection is applied the typical frequency for the $m / n=1 / 1$ mode oscillation is above $10 \mathrm{kHz}$, corresponding to toroidal rotation velocities $v_{\text {tor }}>110 \mathrm{~km} / \mathrm{s}$. With the counter neutral beam injector this frequency can be reduced below $1 \mathrm{kHz}$.

The main diagnostics used for the analysis of MHD activity were

(a) A set of ECE radiometers measuring the electron temperature profile and temperature perturbations due to mode activity in the second harmonic, or, when the electron density reached the cut-off value, an additional set of four radiometers at the third harmonic.

(b) Twelve poloidally and eight toroidally arranged Mirnov coils were used for detection of mode numbers.

(c) The electron density profile and the plasma current distribution were measured with the $\mathrm{HCN}$ interferometer/polarimeter.

(d) Information on the toroidal rotation of the plasma and ion temperature was provided by charge exchange recombination spectroscopy.

(e) The radiated power density was determined by a 34 channel bolometer.

\section{Experimental results and discussion}

\subsection{Confinement back-transitions}

A typical example of an RI mode discharge at the $\beta$ limit is shown in Fig. 1. After application of additional heating at 0.6 and $0.8 \mathrm{~s}$ the feedback controlled seeding of neon is switched on at $t=1.2 \mathrm{~s}$. As a consequence the radiated power increases. Confinement improves as can be seen on the traces of the diamagnetic energy, and the confinement quality reaches a value of 1 with respect to ELM-free $\mathrm{H}$ mode confinement. The line averaged electron density increases to the Greenwald density indicated by the horizontal dashed line. The Greenwald density is defined by

$\bar{n}_{e, G}=10^{20}\left[(\mathrm{MA})^{-1} \mathrm{~m}^{-1}\right] \kappa \bar{j}$

where $\kappa$ is the elongation of the plasma and $\bar{j}$ the average plasma current density [11]. At $t=2.3 \mathrm{~s}$ a sudden decrease of the stored energy (and the plasma pressure) occurs. The heating power as well as the radiated power fraction remain unchanged. The confinement has undergone a transition back to L mode confinement quality seen in the drop of $f_{H 93}$, the ratio of the energy confinement time divided by the 


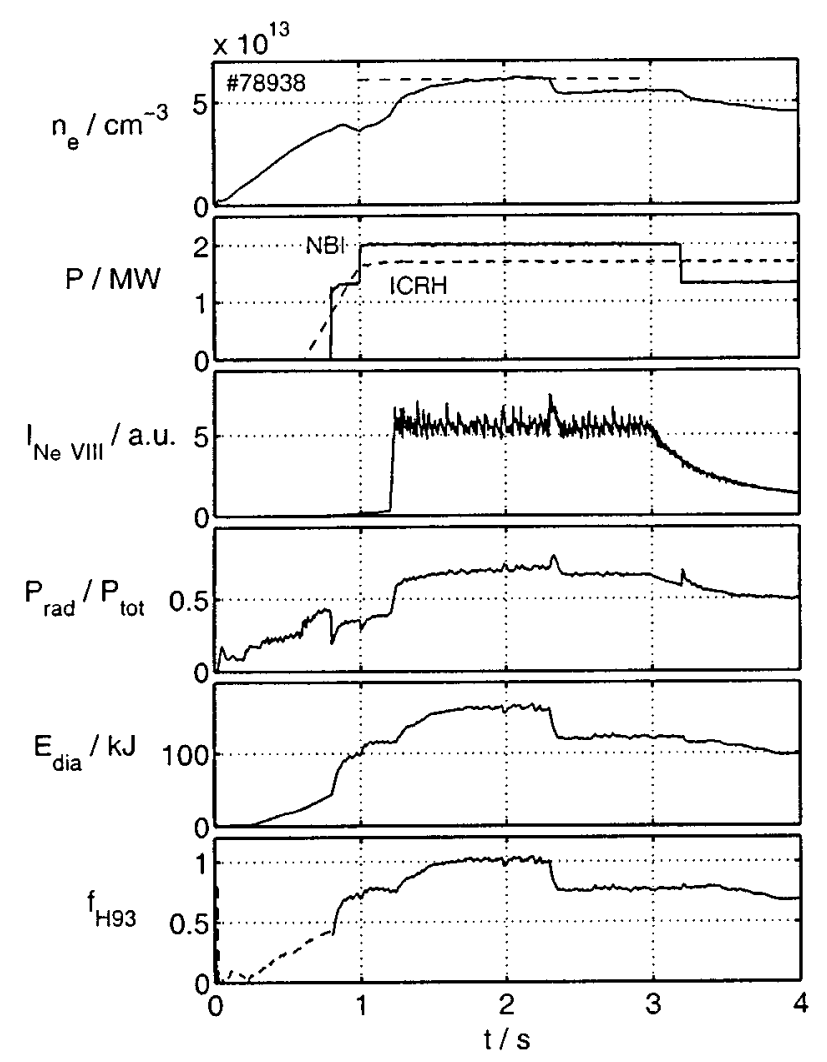

Figure 1. Example of an RI mode discharge where at $t=2.3 \mathrm{~s}$ a confinement back-transition occurs (TEXTOR-94 discharge 78938). The traces from top to bottom display the line averaged electron density, the additional heating power by NBI and ICRH, the brightness of an Ne VIII line, the ratio $\gamma=P_{\text {rad }} / P_{t o t}$ of the radiated power to the total heating power, the diamagnetic energy and the enhancement factor of the confinement with respect to ITER-93 $\mathrm{H}$ mode scaling. Note that the curve for $f_{H 93}$ is meaningless before the start of auxiliary heating because the ITER-93H scaling law does not apply for ohmic plasmas.

ITER-93H scaling law for ELM-free $\mathrm{H}$ mode plasmas [12], down to about 0.7. These confinement backtransitions are correlated to the onset of core MHD activity as will be shown later.

\subsection{Scaling of plasma pressure}

In Fig. 2(a) normalized $\beta$ is plotted versus the socalled Greenwald number,

$N_{G}=\bar{n}_{e} / \bar{n}_{e, G}$

i.e. the line averaged electron density normalized to the Greenwald density limit. The data shown in this figure are taken from the RI mode database and (a)

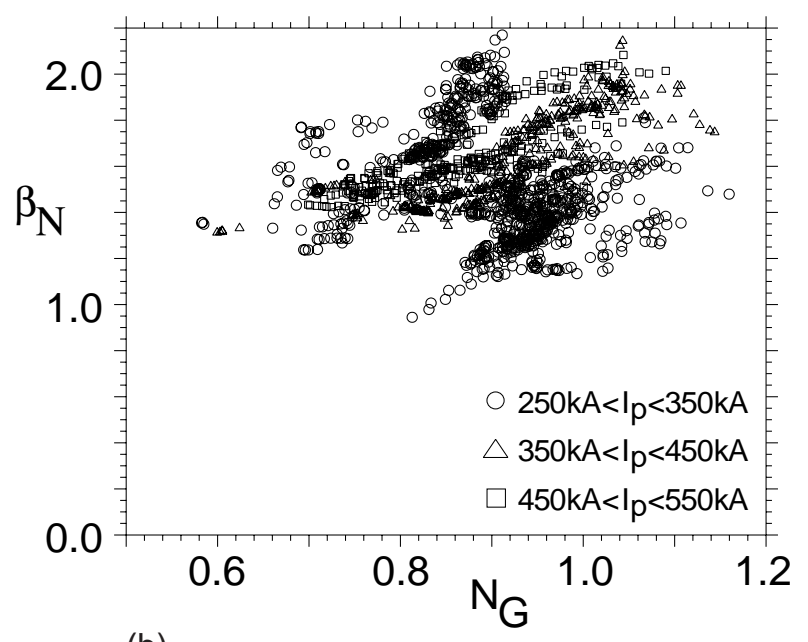

(b)

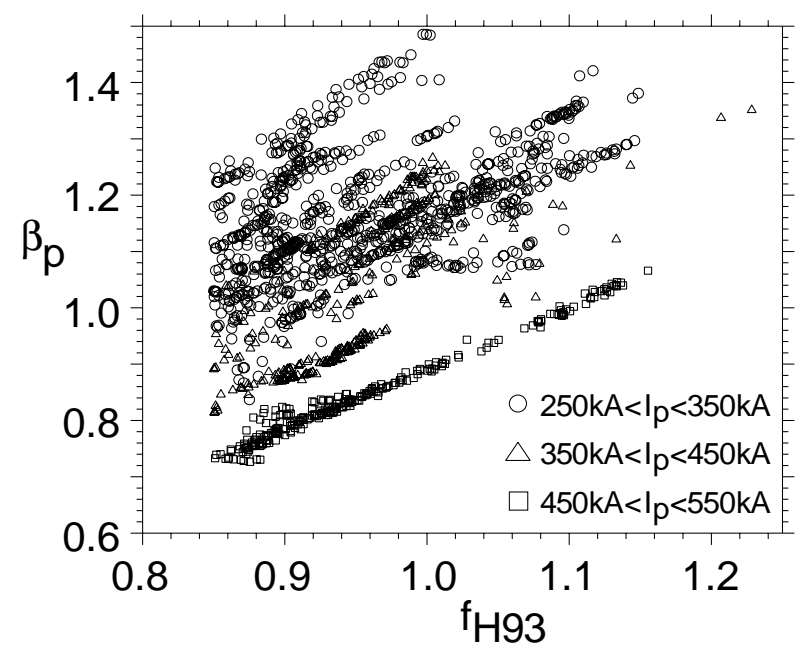

Figure 2. (a) Scaling of $\beta_{N}$ values achieved with Greenwald number (i.e. the line averaged electron density normalized to the Greenwald density limit). (b) Scaling of $\beta_{p}$ with the confinement enhancement factor $f_{H 93}$. Only points which have a confinement equal to or exceeding ELMy $\mathrm{H}$ mode scaling have been selected from the database. The data are collected from 50 discharges; a total of 340 time frames are plotted. The individual samples were averaged over $30-300 \mathrm{~ms}$, i.e. approximately $1-10$ energy confinement times.

represent stationary as well as transient discharge conditions. The maximum value for $\beta_{N}$ reached in TEXTOR-94 is 2.2. The highest values are obtained at electron densities around the Greenwald density because the RI mode confinement time scales proportional to the line averaged electron density

$\tau_{R I}=0.18 \bar{n}_{e} P^{-2 / 3}$ 


\section{H.R. Koslowski et al.}

(where the units are: $\mathrm{s}, 10^{20} \mathrm{~m}^{-3}$, MW) and continues the linear ohmic confinement (LOC) scaling into the range of high densities [13].

The scaling of poloidal $\beta$ with confinement enhancement factor is shown in Fig. 2(b). The data points are marked according to the plasma current. It is clearly visible that the highest $\beta_{p}$ values are attained at low plasma currents, whereas Fig. 2(a) illustrates that the limit in normalized $\beta$ is attained at high as well as at low plasma currents.

At the upper limits in Figs 2(a) and (b) a deterioration of confinement and a drop or saturation of $\beta$ occur. A correlation with the onset of MHD activity in the plasma core is observed, when a confinement back-transition, as described in Section 2.1, takes place. More details on this finding will be discussed in the following. There are in addition discharges where a slow rollover of confinement quality can be observed. In these specific cases no clear correlation with the onset of MHD activity can be found, but it cannot be excluded that the limited time resolution of the diagnostics does not allow a detection of modes with a high frequency. A common observation near the $\beta$ limit is a change in the regular sawtooth activity, which becomes irregular or even completely stabilized. The $m=1$ mode is still present and the mode amplitude and duration can be enhanced.

\subsection{Stabilization of sawteeth and start of MHD mode activity}

Another example of a confinement back-transition observed near the $\beta$ limit is shown in Fig. 3. In the upper part (a) the stored energy as measured by the diamagnetic loop is plotted. At $t=2.0 \mathrm{~s}$ and $t=2.3 \mathrm{~s}$ a sudden decrease in energy content is visible. Part (b) shows the axial value of the current density measured by FIR polarimetry [14]. During the time interval shown a constant decrease in central current density, indicating a broadening of the plasma current distribution, is observed. This kind of change in current profile is often observed as a precursor to a deterioration of energy confinement [15].

Figure 3(c) displays time traces of the line integrated electron densities measured with the $\mathrm{HCN}$ interferometer along three vertical chords. The magnetic axis is positioned at $R_{m a g} \simeq 1.82 \mathrm{~m}$. When neon seeding is applied at $t=1.3 \mathrm{~s}$ the sawtooth activity in the plasma centre is still present. The energy content and the electron density increase as

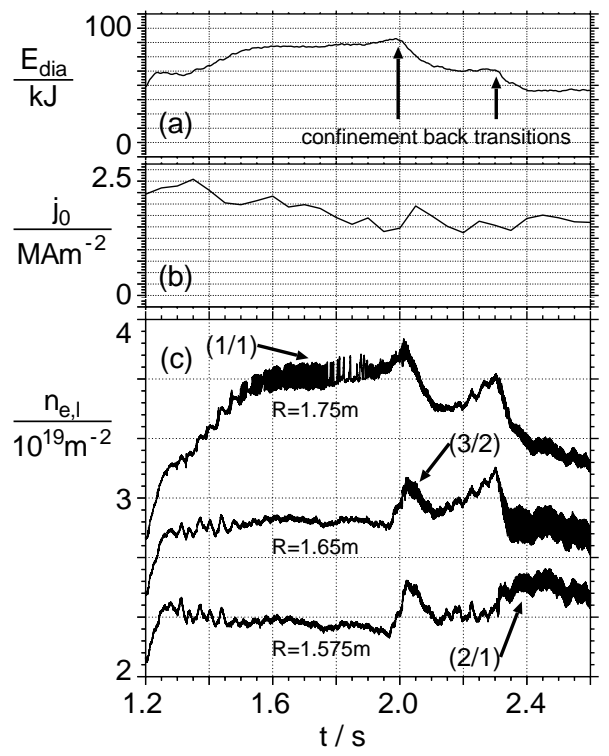

Figure 3. Time traces of (a) diamagnetic energy, (b) current density on axis and (c) line integrated electron densities at three different radial positions for discharge $75203\left(I_{p}=290 \mathrm{kA}\right)$. At $t=2.0 \mathrm{~s}$ and $t=2.3 \mathrm{~s}$, MHD modes with mode numbers $m=3, n=2$ and $m=2$, $n=1$, respectively, set in. Strong $m=1$ modes are visible before the drop in $\beta$.

can be seen from the signal traces of the energy and the interferometer. Later in the discharge the sawtooth activity is stabilized, but mode oscillations with poloidal and toroidal mode numbers $m=n=1$ are visible on the interferometer channel at $R=$ $1.75 \mathrm{~m}$, which passes through the $q=1$ surface. This central $m=1$ mode does not lead to a decrease in stored energy. Even a slight increase of stored energy due to shrinking of mode amplitude is visible just before the first confinement back-transition at $t=2.0 \mathrm{~s}$. The first $\beta$ drop reduces the stored energy by about $25 \%$. Whereas the $m=1$ mode is stabilized, the interferometer channel at $R=1.65 \mathrm{~m}$ detects a second mode oscillation which is located outside of the $q=1$ surface and presumably has mode numbers $m=3, n=2$.

The second transition which reduces the stored energy by an additional $25 \%$ occurs at $t=2.3 \mathrm{~s}$. A saturated $m / n=2 / 1$ mode oscillation with a large amplitude appears (see interferometer channel at $R=1.575 \mathrm{~m}$ ). This second transition is observed in a few cases and does not generally occur. The confinement quality is already degraded when this second transition occurs. We show this example for completeness only. The physical mechanism driving 
(a)

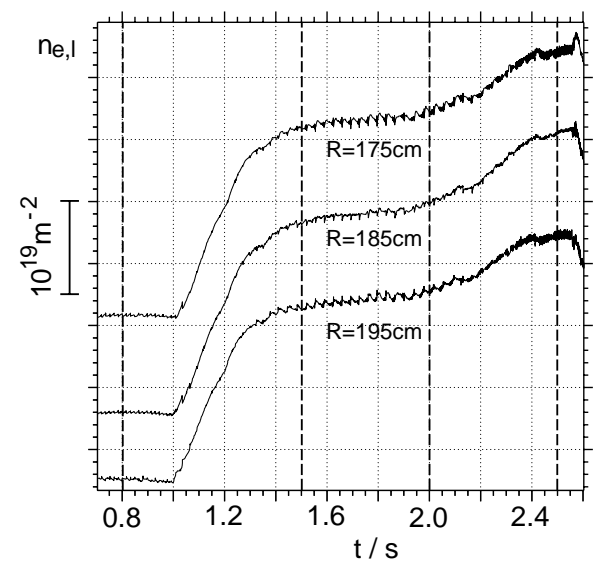

(b)

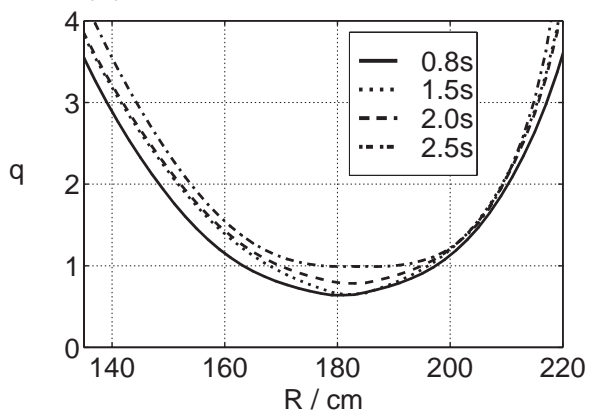

Figure 4. (a) Time traces of line integrated electron densities at various radial positions (discharge 75667). (b) $q$ profiles at different times during the discharge as indicated in part (a) by the vertical dashed lines.

the second mode may be different from the mechanism destabilizing the first mode oscillation. In the following we will focus our investigations on the first transition, which terminates high confinement in the RI mode phase.

The excited modes do not immediately vanish after the drop in plasma pressure, but saturate at a finite amplitude. Once it is excited the $m / n=3 / 2$ mode can last for the rest of the discharge, i.e. it is still present in the ohmic shutdown phase when auxiliary heating has been switched off, although the mode amplitude is decreased.

\subsection{Plasma profile changes}

The profile of the safety factor $q$ has been measured during the evolution from a normal sawtoothing discharge into a state with stabilized sawteeth and pronounced $m=1$ mode activity. The results are displayed in Fig. 4. The upper part (a) shows for reference the time traces of the three channels of the HCN interferometer. For the times marked by (a)

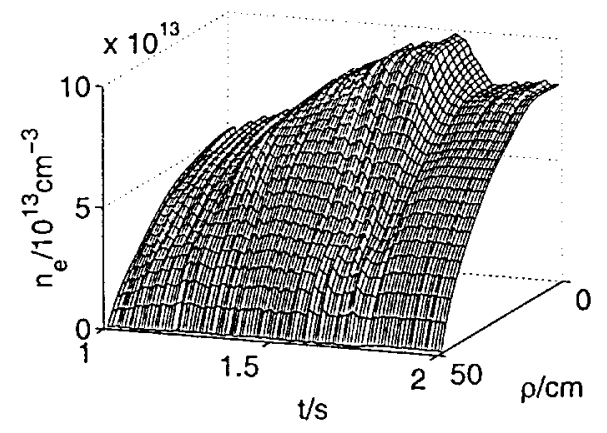

(b)

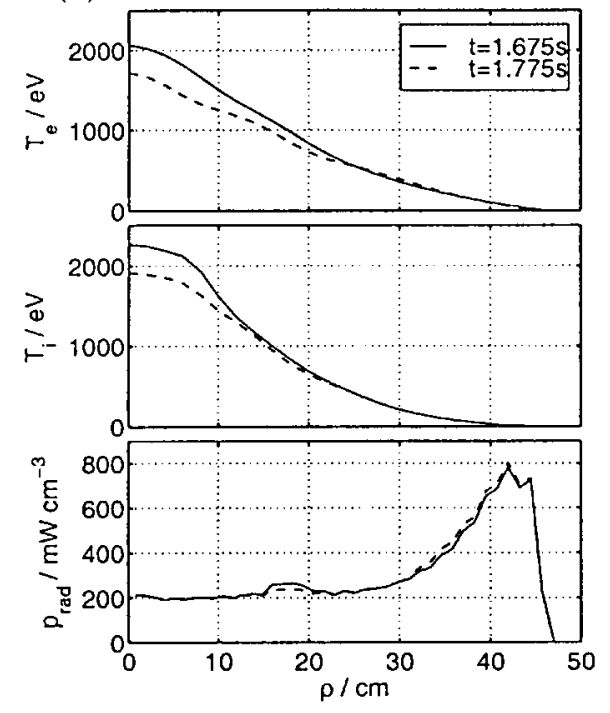

(c)

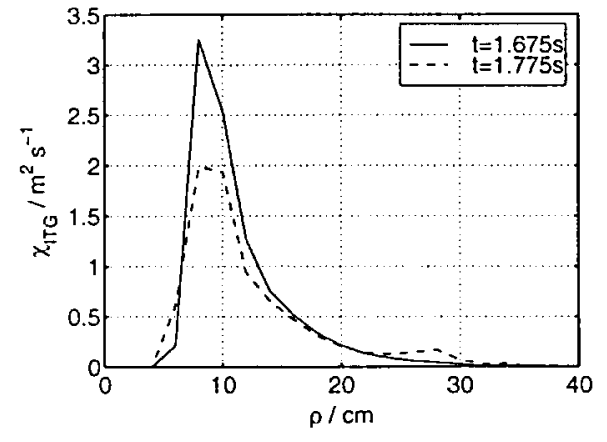

Figure 5. Profiles for discharge 78946 (similar to discharge 78938 shown in Fig. 1). (a) Temporal evolution of the electron density profile plotted versus minor radius $\rho$. The confinement back-transition is at $t=1.7 \mathrm{~s}$. (b) Electron temperature, ion temperature and radiation power profiles before (solid curves) and after (dashed curves) a confinement transition. (c) Heat diffusivity corresponding to ITG turbulence calculated for the profiles just before (solid curve) and after (dashed curve) the backtransition. 


\section{H.R. Koslowski et al.}

vertical dashed lines the $q$ profiles have been evaluated and are plotted in part (b). The central $q$ value remains below unity during the whole phase. The safety factor profile broadens and as a consequence the shear in the central part of the plasma decreases. At intermediate radii the slope of the $q$ profile is rather unchanged.

Figure 5(a) shows the evolution of the electron density profile before and after the $\beta$ drop. The profile is rather peaked with a central value of about $1 \times 10^{20} \mathrm{~m}^{-3}$. When at $t=1.7 \mathrm{~s}$ the $m / n=3 / 2$ MHD mode starts the electron density falls by about $20 \%$. In Fig. 5(b) the profiles of electron and ion temperature as well as the radiated power density before (solid curves) and after (dashed curves) the confinement drop are displayed. Similar to electron density the central temperature values are reduced, but the outer parts of the profiles are not altered after the onset of mode oscillation. The radiated power density shows no change at all.

\subsection{Enhancement of energy transport due to mode activity}

It has been shown previously that the increase of confinement in radiative modes can be attributed to a stabilization of ion temperature gradient (ITG) mode turbulence in the outer part of the plasma [16] due to the modification of the density and temperature profiles by edge radiation. In order to test whether the changed profiles have an influence on this stabilization the heat diffusivity corresponding to ITG turbulence has been calculated before and after the confinement back-transition. The results are shown in Fig. 5(c). As a consequence of the reduced profile gradients after the drop in $\beta$ the ITG mode stabilization in the centre becomes even larger. Within the error bars of the underlying measurements no alteration occurs at medium radii. The enhanced transport observed after the confinement back-transition must therefore be due to sustained mode activity.

\subsection{Theory of neoclassical tearing modes}

Here we briefly review the theory of NTMs in order to establish which characteristics should be looked for to identify whether or not neoclassical effects are responsible for the MHD activity in TEXTOR-94. The growth of tearing modes is described by the generalized Rutherford equation (see for example Ref. [2] and references therein):

$$
\begin{aligned}
\frac{\tau_{r e s}}{r_{m / n}} \frac{d w}{d t}= & f(w)=r_{m / n} \Delta^{\prime}(w) \\
& +r_{m / n} \beta_{p}\left[a_{2} \epsilon^{1 / 2} \frac{L_{q}}{L_{p}} \frac{w}{w^{2}+w_{0}^{2}}\right. \\
& \left.-a_{4}\left(\rho_{p, i} \frac{L_{q}}{L_{p}}\right)^{2} g(\epsilon) \frac{1}{w^{3}}\right]
\end{aligned}
$$

In addition to the well known tearing parameter $\Delta^{\prime}$, additional pressure driven contributions are included. The term proportional to $a_{2}$ contains the effect from the perturbed bootstrap current in the island and is destabilizing, while the term proportional to $a_{4}$ is stabilizing and is due to the polarization current resulting from the drift of the ions when the island moves in the plasma [17]. The quantities in the equation are defined as follows: $\tau_{\text {res }}=$ $\mu_{0} r_{m / n}^{2} /(1.22 \eta)$ is the resistive timescale, $r_{m / n}$ is the radius of the rational surface of the mode, $w$ is the island width, $\epsilon=r_{m / n} / R_{0}$ is the inverse aspect ratio of the resonant surface, $\rho_{p, i}$ is the poloidal ion gyroradius, $a_{2}$ and $a_{4}$ are constants, $L_{p}=p / p^{\prime}$ and $L_{q}=q / q^{\prime}$ are the gradient scale lengths of the pressure and safety factor profiles, $w_{0} \propto\left(\chi_{\perp} / \chi_{\|}\right)^{1 / 4}$ gives a threshold island width below which the flattening of the profiles and therefore the loss of bootstrap current is reduced. This quantity depends on the relation between parallel and perpendicular transport. The function $g$ in the polarization term depends on the ion collision frequency normalized to the diamagnetic drift frequency:

$g\left(\epsilon, \nu_{i i}\right)= \begin{cases}\epsilon^{3 / 2}, & \nu_{i i} /\left(m \epsilon \omega_{e}^{*}\right) \ll C \\ 1, & \nu_{i i} /\left(m \epsilon \omega_{e}^{*}\right) \gg C .\end{cases}$

The constant $C$ is of $O(1)$. At low collisionalities the stabilization of tearing modes by the polarization term is diminished by a factor $\epsilon^{3 / 2}$. A remarkable feature of neoclassical tearing modes arising from the abovementioned formula is that the modes are stable as long as $\Delta^{\prime}<0$ and $w<w_{\text {crit }}$, i.e. the growth of the mode requires a minimum island size. This so-called seed island is created by MHD activity such as sawtooth oscillations, fishbones or ELMs. Once the neoclassical modes are excited they grow to their saturated island size. With falling plasma pressure the modes do not immediately vanish but exhibit a hysteresis. 


\subsection{Analysis of the observed MHD modes}

The above examples have clearly demonstrated the connection between the occurrence of MHD activity and the confinement properties of the plasma. The described sequence from a sawtoothing plasma with high energy confinement into a state with rather poor confinement and strong mode activity is characteristic of many cases where confinement back-transitions have been observed.

In the cases where the deterioration in confinement is due to the onset of an MHD mode the timescale for the drop in stored energy is comparable to the energy confinement time. The cause of the drop in $\beta$ is not always so evident as in the above examples. A slow rollover of plasma energy has been observed on some occasions, when no clear signs of mode activity could be detected. In addition, there are some discharges which show this sudden loss of stored energy but which do not allow an unambiguous identification of destabilized MHD modes. This might be due to a too slow sampling of the data acquisition in these cases, but we cannot exclude completely that other physical effects could be responsible for the confinement loss. In the following discussion we will concentrate on the most common observation of the onset of localized MHD modes with poloidal and toroidal mode numbers $m=3$ and $n=2$, respectively.

\subsubsection{Growth rate of the observed modes}

A comparison between the signals of the $\mathrm{HCN}$ interferometer and the measurements of magnetic perturbation detected by the arrays of Mirnov coils is presented in Fig. 6. The upper part (a) shows central line averaged electron density and part (b) shows normalized beta $\beta_{N}$. At $t=1.7 \mathrm{~s}$ a confinement back-transition occurs and the plasma pressure is reduced by $25 \%$. The reduction takes place within a time of $50 \mathrm{~ms}$, which is approximately equal to the energy confinement time under these conditions. The time-frequency spectra in parts (d) and (e) where the Fourier components are analysed in a small time window and plotted versus time show that the interferometer clearly detects the odd- $m$ mode. The magnetic signal in part (d) is generated as the difference between two suitably chosen coils from the poloidal Mirnov array, thus emphasizing the odd- $m$ signal components. In addition, the RMS value of this signal is plotted in Fig. 6(c). Unfortunately this signal is somewhat perturbed due to pick-up from the vertical field coils which are pulsed with a frequency

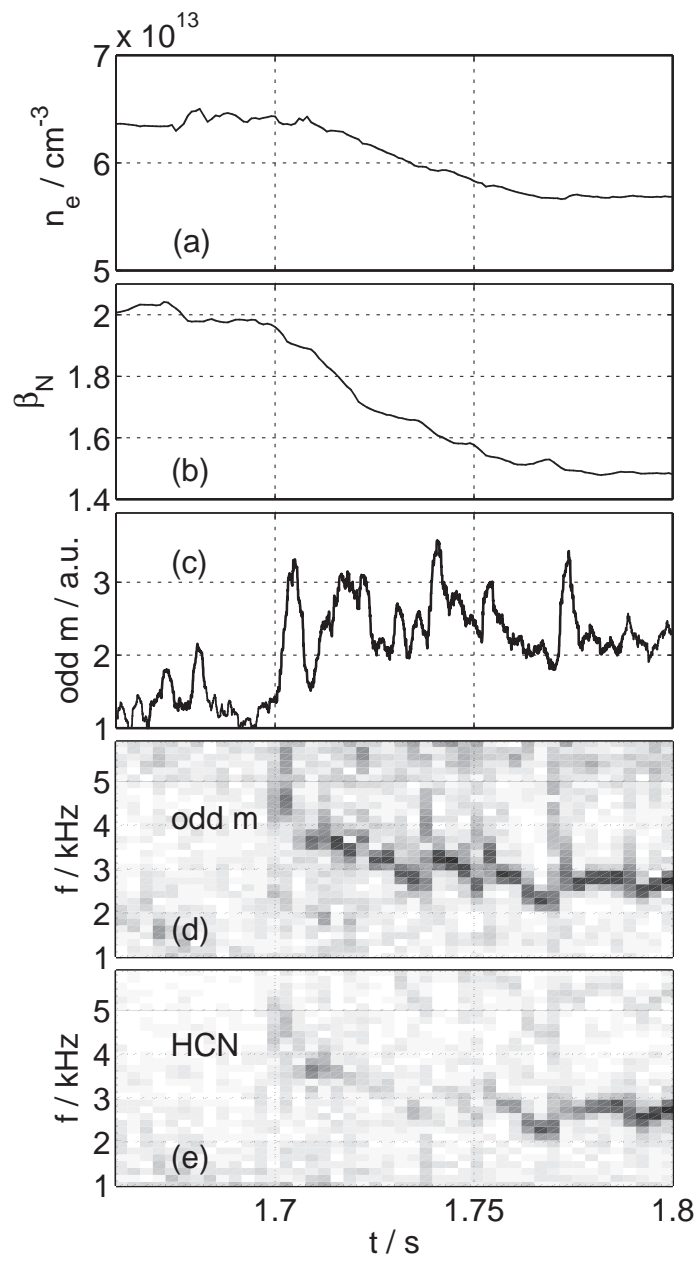

Figure 6. Time traces of (a) the line averaged electron density, (b) the normalized toroidal beta $\beta_{N}$, (c) the RMS amplitude of a combination of two Mirnov coil signals giving the odd- $m$ component of the magnetic perturbations, (d) a spectrogram (windowed Fourier analysis) of the odd- $m$ signal and (e) a spectrogram of one interferometer channel for the same discharge as shown in Fig. 5. The confinement back-transition in this discharge is at $t=1.7 \mathrm{~s}$.

around $1 \mathrm{kHz}$. The rise in mode amplitude, i.e. the growth of the island width, is faster in the magnetic observations. This can be understood from the fact that the islands have to reach a finite size before the resultant flattening of the electron density leads to a strong modulation of the interferometer signal. For the determination of the growth time of the mode it is therefore required to analyse the signals from the magnetic diagnostic, although the information from the profile measurements (ECE, interferometer, SXR emission) is valuable in determining the mode numbers and the location of the mode. The growth time 


\section{H.R. Koslowski et al.}

of the $m / n=3 / 2$ mode (determined from several discharges) is in the range 10-20 ms. The resistive growth time for tearing modes,

$\gamma^{-1} \approx \tau_{R}^{3 / 5} \tau_{A}^{2 / 5}$

is about $3 \mathrm{~ms}$ for typical plasma parameters at the rational $q=3 / 2$ surface under RI mode conditions. The time for the island to reach its saturated size is larger and does not disagree with the observations. As has been pointed out in Ref. [2] it seems very difficult, and is therefore rather unlikely, to identify neoclassical effects from the measurement of mode growth times.

\subsubsection{Determination of tearing parameter $\Delta^{\prime}$}

The growth of resistive tearing modes is governed by the so-called tearing parameter $\Delta^{\prime}$, as long as no neoclassical (pressure driven) effects are taken into account. The HCN polarimeter on TEXTOR94 allows measurement of the plasma current density, and hence a determination of $\Delta^{\prime}$. This has been done for several discharges and the results are shown in Fig. 7(a). The time axis for the individual discharges is offset by the time $\left(t_{\text {onset }}\right)$ when the $m / n=3 / 2$ mode starts. The tearing parameter $\Delta^{\prime}$ has been calculated for cylindrical geometry. A stabilizing contribution due to toroidal effects is not included in this calculation.

$\Delta^{\prime}$ is found to be negative and stays approximately constant during the time interval before mode onset. The reconstruction error of the $q$ profile shows up after the determination of $\Delta^{\prime}$, but the traces stay almost constant, i.e. there seems not to be a general trend to increase. The curves reach positive values for $t-t_{\text {onset }}>0$. The time difference between the onset of the mode and the time when $\Delta^{\prime}$ becomes larger than zero is $20-25 \mathrm{~ms}$, i.e. slightly larger than the time needed for the modes to reach their saturated size (Section 2.7.1). The destabilization and growth of the mode is during a phase where $\Delta^{\prime}$ is negative. This is illustrated in Fig. 7(b), where for one discharge shown in Fig. 7(a) the line integrated electron density at $R=1.575 \mathrm{~m}$ and the normalized $\beta$ are plotted. The timescale is the same as in part (a). The time when the drop in $\beta$ occurs is marked by a vertical dashed line (corresponding to $t-t_{\text {onset }}=0$ in Fig. 7(a)). At the same time the MHD mode activity, visible on the interferometer signal, starts.

The poloidal asymmetry due to the developed mode might result in a rather poor estimation of (a)

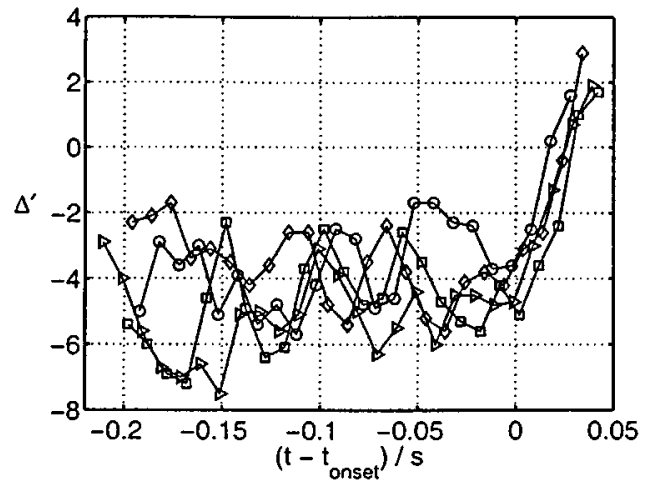

(b)

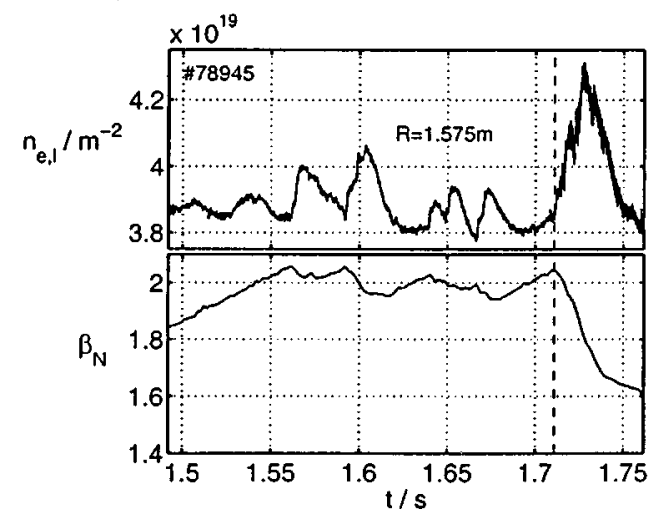

Figure 7. (a) Calculated values for the tearing parameter $\Delta^{\prime}$. The time axis is offset by the time $t_{\text {onset }}$ when the $m=3, n=2$ mode oscillation starts. The four curves correspond to a series of similar discharges (Nos 78938,39,45,46). (b) Line integrated electron density (top) and normalized $\beta$ (bottom) for one discharge from part (a). The time $t_{\text {onset }}$ when $\beta$ drops and the mode activity starts is marked by the vertical dashed line.

the safety factor, as the reconstruction of poloidal magnetic field from FIR polarimetry relies on the assumption of nested circular flux surfaces as well as a constant electron density on these flux surfaces, an assumption violated when strong MHD activity is present. However, the conclusion that the mode grows at negative $\Delta^{\prime}$ has to be taken with some caution, as the island size (a few centimetres) is smaller than the spacing of the probing chords of the polarimeter $(10 \mathrm{~cm})$. The smallest spatial structure which can be detected is larger than the typical island size.

Moreover, if it is true that $\Delta^{\prime}$ becomes positive after the mode has reached saturation, one has to assume that such an increase of the tearing parameter must be compensated for by a decrease of the 
(a)

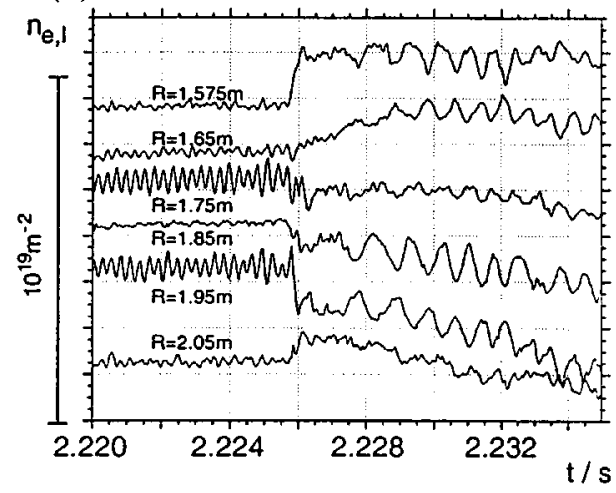

(b)

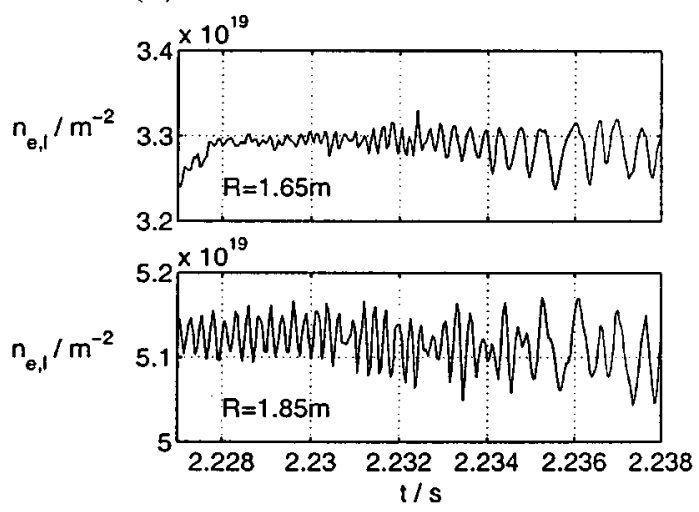

Figure 8. (a) Time traces of six interferometer chords (discharge 77920). At $t=2.226 \mathrm{~s}$ an $m / n=3 / 2$ mode starts after a sawtooth crash. (b) Example of a discharge (No. 75188) where the $m / n=3 / 2$ mode is coupled to the $m=1$ mode oscillation in the centre. The magnetic axis is located at $R=1.87 \mathrm{~m}$.

destabilizing neoclassical term. Altogether the identification of the MHD activity as being an NTM on the basis of a negative $\Delta^{\prime}$ is therefore not very strong.

\subsubsection{Trigger for the $m / n=3 / 2$ mode}

As has been stated earlier, the core MHD activity shows characteristic changes under plasma conditions with high confinement. The sawtooth activity, which is normally present under almost all discharge conditions, is stabilized. The sawteeth are replaced by mode activity at the $q=1$ surface which is similar to the sawtooth precursor oscillations but lasting for longer time periods. Under these conditions the highest $\beta$ values are reached. The $m=1$ modes do not seem to have a strong detrimental influence on the energy content, as this can even increase by about $20 \%$ as compared with the preceding sawtoothing period. The stabilization of the sawteeth is not complete, instead irregular crash events in the core can be observed. This behaviour resembles the so-called $\mathrm{Z}$ mode found on ISX-B [18]. An example is presented in Fig. 8(a), where several traces of the HCN interferometer measured at various radial positions are displayed. The magnetic axis is located at $R=1.82 \mathrm{~m}$. The two channels at $R=1.75 \mathrm{~m}$ and $1.95 \mathrm{~m}$ show the $m=1$ oscillation with opposite phase, indicating an odd poloidal mode number. This oscillation appears in the same channels, i.e. at the same spatial location as the normal sawtooth precursor oscillation at the $q=1$ surface in the previous phase of the discharge. At $t=2.226 \mathrm{~s}$ a sawtooth crash occurs in the core. This can be seen from the rapid decay of the inner channels $(R=1.75 \ldots 1.95 \mathrm{~m})$ and the inverted sawteeth visible at $R=1.575 \mathrm{~m}$ and $R=2.05 \mathrm{~m}$. After this collapse in the core a second mode appears. At the same time the drop in energy confinement starts. This mode is located more outside than the $m=1$ precursor, as can be seen for example by the modulation of the channel located at $R=1.575 \mathrm{~m}$ on the high field side. The rational surface is located in-between the radial positions where the oscillations on adjacent channels have a phase inversion. Again, this mode has an odd poloidal mode number. This is confirmed by the measurements of the Mirnov coils, which show the onset of an odd- $m$ component. From the measurement of the safety factor profile one can conclude that this mode has the mode numbers $m=3$ and $n=2$.

In most of the discharges where confinement deterioration due to the onset of an $m / n=3 / 2$ mode has been observed, a preceding sawtooth crash in the core acts as a trigger for this mode. In some discharges a mode onset before the collapse has been found. In those cases the $3 / 2$ mode is coupled to a strong $m=1$ oscillation. An example of this is shown in Fig. 8(b), where the interferometer channel at $R=1.85 \mathrm{~m}$ measures the $m=1$ mode oscillation in the center. The second channel at $R=1.65 \mathrm{~m}$ shows the development of the $3 / 2$ mode phase locked to the central $m=1$ mode. This behaviour is less frequent than the triggering of the $3 / 2$ mode by a core crash event. Approximately $20 \%$ of the cases investigated show a mode coupling. The majority of the $3 / 2$ modes leading to confinement deterioration is destabilized by a sawtooth crash, which can be interpreted as the origin of a seed island as required by NTM theory. 
(a)

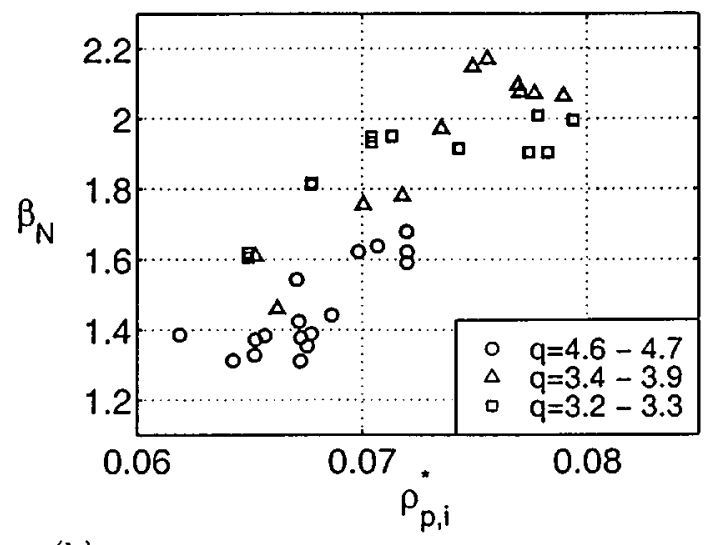

(b)

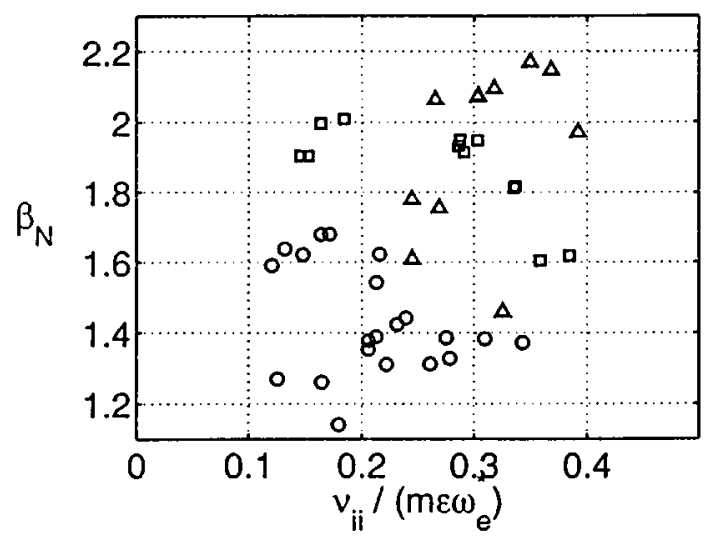

Figure 9. Scaling of $\beta_{N}$ with (a) the poloidal ion gyroradius measured at the mode resonant surface at onset of the mode activity, and with (b) the normalized collisionality. The data points are sorted by edge safety factor.

\subsubsection{Scaling of poloidal $\beta$ with normalized ion gyroradius and normalized collisionality}

The growth of tearing modes due to neoclassical effects depends on the balance between the pressure dependent stabilizing and destabilizing contributions, as long as the tearing parameter is negative. In particular, the stabilization due to the ion polarization current depends on the poloidal ion gyroradius. A plot of the normalized toroidal $\beta$ versus the poloidal ion gyroradius normalized to the plasma radius, $\rho_{p, i}^{*}=\rho_{p, i} / a$, is shown in Fig. 9(a). The poloidal ion gyroradii are measured at the mode resonant surface. The data points are marked with respect to the edge safety factor. This is done in order to keep the profile shapes in the various groups of data points as similar as possible. The achieved normalized $\beta$ increases with $\rho_{p, i}^{*}$. All groups of data points exhibit the same behaviour. Within the error bars $\beta_{N}$ has an approximately linear dependence upon $\rho_{p, i}^{*}$ at lower values of the poloidal ion gyroradius. At higher values of $\rho_{p, i}^{*}$ the data measured at lower $q_{a}$ ( $q_{a}<4$, triangles and squares) show saturation. A similar behaviour has been found at ASDEX Upgrade [19], where this scaling is used to demonstrate the role of the ion polarization current for the stabilization of neoclassical tearing modes.

In addition, some caution with this scaling has to be taken because there exists a collinearity between $\beta_{N} \propto n T$ and $\rho_{p, i} \propto T^{1 / 2}$. One has to expect an increase of $\beta_{N}$ with $\rho_{p, i}$ in any case. A plot of the normalized $\beta$ versus $n \rho_{p, i}^{2}$ shows a saturation at higher values which cannot be explained by the intrinsic dependence of $\beta_{N}$ on the product $n \rho_{p, i}^{2}$.

For comparison with other experiments we show in Fig. 9(b) a plot of $\beta_{N}$ versus the normalized collisionality $\nu_{i i} /\left(m \epsilon \omega_{e}^{*}\right)$. Again the different groups of data points are ordered by the safety factor at the edge. The data have a relatively large scatter which is mainly due to the difficult determination of the profile gradients from the experimental data. It is worth noting that ITER will operate with $\beta_{N}=2-2.5$ at $\nu_{i i} /\left(m \epsilon \omega_{e}^{*}\right)=0.13$. At that normalized collisionality the maximum achieved $\beta_{N}$ in the circularly shaped TEXTOR-94 tokamak reaches values up to 2 .

\subsection{Stationarity of high confinement discharges}

We have demonstrated in the previous sections that the stabilization of sawtooth oscillations may lead to states with the highest confinement $\left(f_{H 93} \approx\right.$ 1.2 ) and that it is difficult to achieve this in a stationary way. In order to reach a long stationary flat-top phase of the discharge the plasma performance has to be slightly reduced below the values where the $\beta$ limit is expected. A discharge with a $f_{H 93}$ value above 0.95 and a flat-top time of $6.8 \mathrm{~s}$, equal to $160 \tau_{E}$, where all plasma parameters were constant, has been achieved. An overview on the main plasma parameters is given in Fig. 10. The discharge parameters were chosen in order to allow a long steady state phase of the discharge. This has been achieved mainly by utilization of the $\beta$ feedback loop which allows adjustment of the ICRH power in real time and keeping the stored energy in the plasma constant. This discharge has regular sawteeth during the whole flat-top phase. 


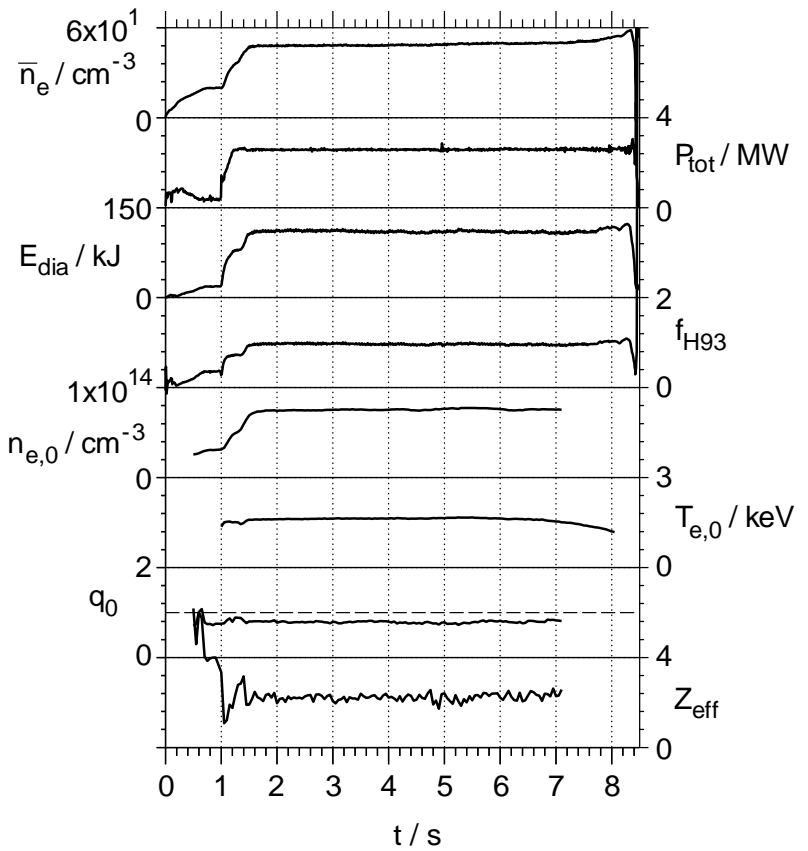

Figure 10. Long stationary discharge with a flat-top duration $\Delta t=6.8 \mathrm{~s}$ (No. 75679). The traces from top to bottom are: the line averaged electron density, which is around the Greenwald density; the total applied heating power; the stored energy; the confinement enhancement factor $f_{H 93}$, the central electron density, which is constant indicating no change of the density profile; the central electron temperature (note that the decay at the end of the discharge is due to a too early switch-off of the toroidal magnetic field, thus the ECE resonance position is shifted to the high field side); the safety factor onaxis, which indicates no temporal change of the current distribution; and, the effective charge determined from resistivity, which exhibits no sign of impurity accumulation.

\section{Summary and conclusion}

The $\beta$ limit of discharges in the RI mode, which is the regime with the highest confinement in TEXTOR-94, has been explored. The normalized $\beta$ reaches values up to 2.2 , while the poloidal $\beta$ is limited below 1.5. Transiently reached beta values are approximately $20 \%$ higher as compared with stationary conditions. Staying slightly below the limits allows stationary discharges with a confinement better than the ELMy $\mathrm{H}$ mode scaling projected for ITER. These discharges have been demonstrated with a flat-top time up to $6.8 \mathrm{~s}$. This corresponds to 160 times the energy confinement time. This is the same relation between burn time and energy confinement time as foreseen for ITER.
The limitation of the maximum achievable plasma pressure under radiative improved mode conditions is correlated with the onset of MHD activity in the plasma. The most dominant mode has been identified as an $m / n=3 / 2$ tearing mode.

The drop in stored energy or plasma pressure due to the tearing mode is about $25 \%$. The stabilization of ITG turbulence in the plasma which is strongly improved by the radiating impurity is not altered, but becomes even better due to the smaller profile gradients. The MHD mode has been held responsible for the plasma pressure drop.

The mode growth shows saturation and the modes reach their saturated sizes within 10-20 ms. The tearing parameter $\Delta^{\prime}$ is found to be negative when the modes are destabilized and reach slightly positive values after the modes become saturated. The modes do not vanish after the drop in plasma pressure and can be observed for the rest of the auxiliary heating phase up to the rampdown of the plasma current. The onset of MHD activity is found to show a correlation with a sawtooth crash. In some cases a coupling of the $m / n=3 / 2$ mode to the $m=1$ mode in the plasma core is found. The achievable normalized beta, $\beta_{N}$, increases with poloidal ion gyroradius and shows saturation at larger values.

The observed phenomenology has some similarities to the observations of NTMs on other tokamak experiments. However, the data available at present do not allow an unambiguous identification of the neoclassical effects responsible for the destabilization of the observed tearing modes. Some results, like the growth at negative $\Delta^{\prime}$ as well as the requirement of a perturbation creating a seed island, seem to confirm neoclassical contributions. On the other hand, the lack of a lower value for the plasma pressure where the excited modes decay (hysteresis behaviour), as well as the increase in the tearing parameter in the phase during which the modes are sustained, point to the MHD modes having an ordinary nature. A possible explanation might be that the mode starts as a NTM, i.e. the destabilization is pressure driven, and the change in the equilibrium afterwards transforms the mode into an ordinary tearing mode (with positive $\Delta^{\prime}$ ) which does not vanish when the plasma pressure drops.

In the future more experiments, which will be aimed at clarifying possible neoclassical effects, will have to be performed. In the case pressure dependent contributions are confirmed, the typical parameter range of the TEXTOR-94 RI mode with high densities and normalized collisionalities $\nu_{i i} /\left(m \epsilon \omega_{e}^{*}\right)$ in the 


\section{H.R. Koslowski et al.}

range from 0.1 up to 0.4 would allow investigation of $\beta$ limiting phenomena in a parameter regime which is relevant for ITER.

\section{References}

[1] Troyon, F., et al., Plasma Phys. Control. Fusion 26 (1984) 209.

[2] Sauter, O., et al., Phys. Plasmas 4 (1997) 1654.

[3] Zohm, H., et al., Plasma Phys. Control. Fusion 39 (1997) B237.

[4] La Haye, R.J., et al., in Fusion Energy 1996 (Proc. 16th Int. Conf. Montreal, 1996), Vol. 1, IAEA, Vienna (1997) 747.

[5] Chang, Z., et al., Phys. Rev. Lett. 74 (1995) 4663.

[6] Isayama, A., Kamada, Y., Ozeki, T., Isei, N., Plasma Phys. Control. Fusion 41 (1999) 35.

[7] Gude, A., Günter, S., Sesnic, S., ASDEX Upgrade Team, Nucl. Fusion 39 (1999) 127.

[8] Wolf, G.H., et al., in Fusion Energy 1996 (Proc. 16th Int. Conf. Montreal, 1996), Vol. 1, IAEA, Vienna (1997) 177.

[9] Ongena, J., et al., Plasma Phys. Control. Fusion 38 (1996) 279.
[10] Jaspers, R., et al., in Controlled Fusion and Plasma Physics 1998 (Proc. 25th Eur. Conf. Prague, 1998), Vol. 22C, European Physical Society, Geneva (1998) 552.

[11] Greenwald, M., et al., Nucl. Fusion 28 (1988) 2199.

[12] Thomsen, K., et al., Nucl. Fusion 34 (1994) 131.

[13] Weynants, R.R., et al., Nucl. Fusion 39 (1999) 1637.

[14] Koslowski, H.R., Soltwisch, H., Fusion Eng. Des. 34\&35 (1997) 143.

[15] Koslowski, H.R., et al., Plasma Phys. Control. Fusion 39 (1997) B325.

[16] Tokar', M., et al., Plasma Phys. Control. Fusion 41 (1999) L9.

[17] Wilson, H.R., Conner, J.W., Hastie, R.J., Hegna, C.C., Phys. Plasmas 3 (1996) 248.

[18] Lazarus, E.A., et al., Nucl. Fusion 25 (1985) 135.

[19] Günter, S., et al., Nucl. Fusion 38 (1998) 1431.

(Manuscript received 23 June 1999

Final manuscript accepted 27 January 2000)

E-mail address of H.R. Koslowski:

H.R.Koslowski@FZ-Juelich.de

Subject classification: B0, Te; C0, Te; D2, Te;

D3, $\mathrm{Te}$ 